\title{
Dynamic response of continuous beams with discrete viscoelastic supports under sinusoidal loading
}

\author{
Bing $\mathrm{Li}^{1}$, Shaohua Wang ${ }^{1 *}$, Xiao $\mathrm{Wu}^{1}$, Bin Wang ${ }^{1,2^{*}}$ \\ ${ }^{1}$ School of Mechanical Engineering, Southwest Jiaotong University, China \\ ${ }^{2}$ School of Engineering and Design, Brunel University, UK
}

\begin{abstract}
Analysis of vibrations of continuous beams with discrete viscoelastic supports has been established through theoretical modeling and a finite element analysis. The theoretical model is based on the Euler-Bernoulli theory, and the Ritz approach was employed to obtain numerical results from which the attenuation of the beam's vibration was obtained. In parallel, a finite element analysis was carried out in ABAQUS using 3D beam elements. It is shown that the results of theoretical calculation agree well with those of the finite element analysis.

Both models were applied to explore geometric and design variations, and then to a full model of a bridge expansion unit as an application example. The vibration of the beams in the design, the influence of the stiffness and the viscous damping coefficient of the supports were discussed, demonstrating the models' usefulness in helping with design optimization.
\end{abstract}

Keywords: vibration, beams, finite element analysis, viscoelastic support, bridge expansion units

*Corresponding authors: wangsh1963@tom.com; bin.wang@brunel.ac.uk 


\section{Introduction}

Expansion units and joints are commonly used in bridges or viaducts to accommodate temperature-induced movements between bridge decks and abutments. The functions of the units are twofold: they need to limit the internal stresses due to thermal expansion under high temperatures which may cause buckling failure of the long-span beam decks; and to minimize gaps caused by shrinkage under low temperatures for smooth traffic flows over the bridge [1-3]. With the development of motorways, city road viaducts and elevated high speed railway lines, more stringent requirements have been placed on the development of bridge expansion units to allow for large gaps/displacements. Such expansion units are used in hostile environmental conditions and loaded heavily by high volume traffics. The maximum gap between contiguous beams can now reach to $80 \mathrm{~mm}$ [4-6]. Fatigue failure under repeated impact is the main cause of damage to bridge/viaduct structures. Apart from the required strength, the expansion units also need to be of low costs, can be installed easily, and require minimum maintenance with long durability.

Fig. 1 shows an example of a module design of a bridge expansion unit. Crossbeams are laid in parallel in the direction of the axis of the bridge underneath the bridge deck and are allowed to expand/shrink freely through the use of sliding bearings. They support a number of beams (here named I-beams, as being used in the design and to differentiate with the crossbeams) crossly laid on top of cross-beams and leveled to the surface of the deck with predetermined equal distance/gaps amongst the I-beams. The number and the size of the I-beams required are determined 
by the overall deck gap the expansion unit needs to accommodate. And the number of the crossbeams needed depends on the width of the bridge.

With the temperature effect on the crossbeams, the supported I-beams move with the crossbeams to mitigate the gaps amongst them. When designed properly, this mitigation movement will enable the gaps between the I-beams to remain within the required range under all weather conditions to reduce the impact loading caused by the traffic over the gaps.

The dynamic response of the beams (both the I-beams and the crossbeams) needs to be analyzed for potential structural damages [7]. Wang [8] did fatigue tests on several modular expansion units, showing that the residual stress in the beams increases with the number of load cycles. Dexter et al [9] did study on the structural design, installation and maintenance of expansion joints, suggesting that elastomeric parts and fasteners are best addressed through performance tests on the modular joint unit as a system. Coelho et al did the dynamic tests of modular expansion joints [10-11]. They showed that the traffic speed has influence on the strain distribution along the centre beam, and the design of the modular joint systems must pass both static and dynamic performance tests. Michael [12] established the load form and a theoretical model of the lamella beam-grid expansion joint, and concluded that the dynamic amplification is important for design, and in some cases, its value is higher than those prescribed in the current design codes. Roeder [13] studied the fatigue of modular expansion joints, and showed the importance of the load spectrum on the fatigue life of expansion units. Chaallal $\mathrm{O}$ analyzed the results of fatigue tests and provided detailed stress 
distributions. Crocetti R [15] proposed a design load approach through fatigue tests. Ghimire JP [16] studied the noise generation and radiation from a modular expansion joint. These studies were focused on the performance of whole joint units, where understanding on the responding mechanism of the individual beams and the influence on the viscous supports is weak.

For the purpose of strength and fatigue analysis, the design of the expansion unit can be approximately modeled as continuous elastic beams with discrete viscoelastic supports. This is applicable to both the I-beams on top, and the crossbeams beneath. Most of the studies on beam with elastic foundations are for continuous, non-interrupted supports, such as those in [17-20] where different material models and loading conditions are considered. Yu and his colleagues did dynamic analysis of impact loading on beam-on-foundation based on a simplified rigid-plastic model [17]. Chen and others studied the elastoplastic beam-on-foundation model, mainly on the quasi-static behavior [18-19]. Zhou et all studied the elastic behavior of ring-on-foundation [20]. However, analyses on beams under discrete viscoelastic supports are rare.

In this paper, we present an analytical solution for the response of beams on discrete viscoelastic supports under dynamic loading. Numerical simulations using finite element code ABAQUS were also obtained and compared with the theoretical model results, through which, the mechanical properties of the system are analyzed for design purpose. 


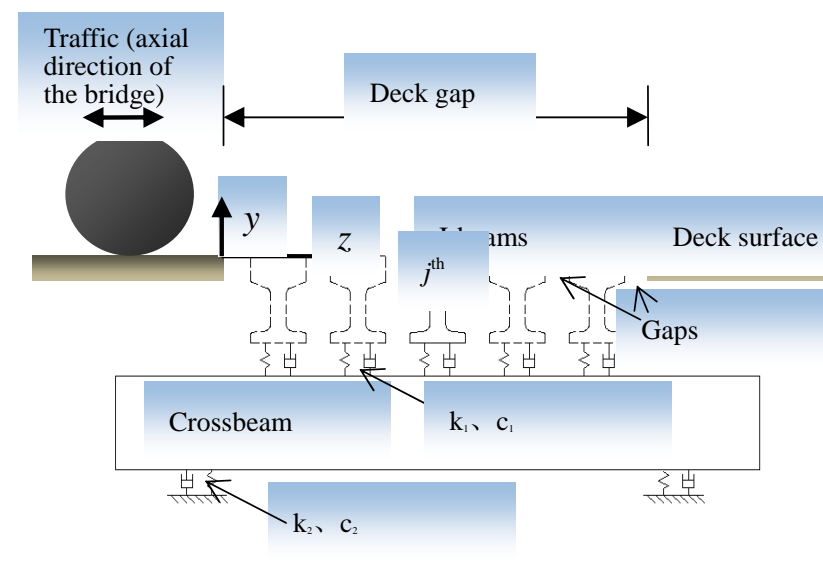

(a) bridge side view

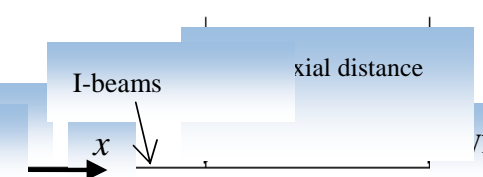

Theel pressure

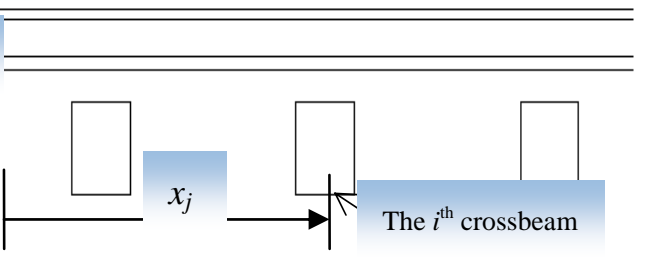

(b) traffic direction view

Fig.1. A modular design of an expansion joint composing of 5 I-beams perpendicular to the traffic supported by a number of crossbeams aligned to the axis of the bridge

\section{Dynamic equations}

For continuous beams, transverse vibrational equations can be obtained by the Euler-Bernoulli theory. In Fig. 1, $z$ represents the axial direction of the bridge, ie. the traffic direction, $y$ the vertical downwards direction and $x$ the direction of the bridge width. Let $y_{I j}(x, t)$ denote the vertical displacement of the $j^{\text {th }}$ I-beam, where the subscript $I$ indicates the I-beam. The vibration equation of the I-beam is given by [21]

$$
E I_{1} y_{I j}^{(4)}(x, t)+m_{1} \ddot{y}_{I j}(x, t)=-\sum_{i=1}^{N I} F_{c z}(t) \delta\left(x-x_{i}\right)+P(x, t)\left[\delta\left(x-x_{r}\right)+\delta\left(x-x_{l}\right)\right]
$$

where $E I_{1}$ is the flexural rigidity of the I-beam. The superscript (4) represents the $4^{\text {th }}$ order derivative with respect to time, $m_{1}$ the mass per unit length, $F_{c z}$ the supporting force by the crossbeams underneath. NI is the total number of supports to the I-beam or the number of the crossbeams in the expansion unit, and $x_{i}$ the position of the $i$ th support. $P$ is the impact force from the traffic with subscripts $r$ and $l$ representing the 
right and left wheels of the vehicle. $\delta$ is the Kirchhoff function.

$$
\delta\left(x-x_{i}\right)=\left\{\begin{array}{cc}
0 & x \neq x_{i} \\
1 & x=x_{i}
\end{array}\right.
$$

In modern bridge design $[1,16]$, the standard axle load is considered $140 \mathrm{kN}$ up to a velocity of $100 \mathrm{~km} / \mathrm{h}$. The contact area of a wheel and the road surface is assumed to be $0.2 \mathrm{~m}$ by $0.4 \mathrm{~m}$, and the axial distance between the two wheels is taken as $1.8 \mathrm{~m}$, as shown in Fig.2. For the most unfavorable condition, the wheels are assumed loading at the mid span between neighboring supports as shown in Fig. 1(b). The dynamic load pulse of a wheel can be described by a sine wave as shown in Fig. 3(a) [12]. The time period of the half wave depends on the traffic speed.

Let $P(x, t)$ be the pulse loading of one wheel, we assume

$$
P(x, t)=-\frac{P_{n}}{2}[1+\sin (\omega t-\pi / 2)]
$$

where $P_{n}$ is the weight loading of a vehicle and remains constant. The velocity dependent impact effect will be considered later by an impact coefficient (in Section 4.3).

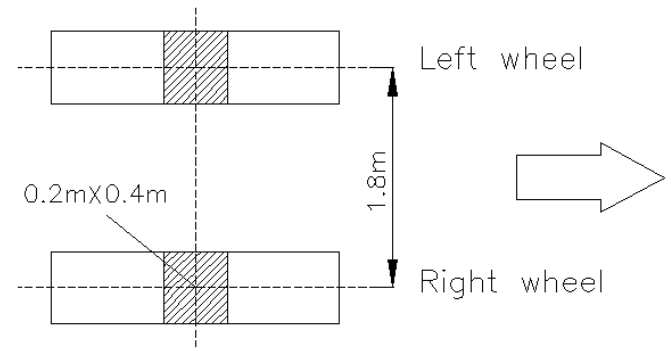

Fig.2. Geometrical description of wheel contact 


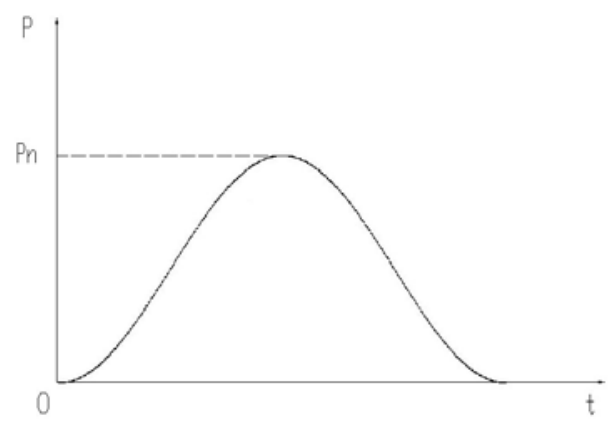

(a)

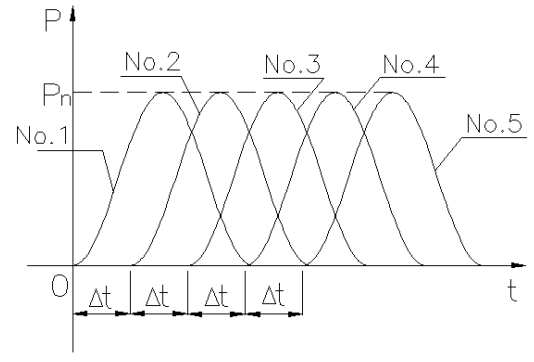

(b)

Fig.3. Dynamic loading (a) Load pulse to an I-Beam, (b) Time sequence of dynamic loading over a 5-I-beam system

For the supporting forces received by the I-beam, we can obtain.

$$
-\sum_{i=1}^{N I} F_{c z}(t) \delta\left(x-x_{i}\right)=\sum_{i=1}^{N I} k_{1}\left[y_{c i}\left(x_{j}, t\right)-y_{I j}\left(x_{i}, t\right)\right]+\sum_{i=1}^{N I} c_{1}\left[\dot{y}_{c i}\left(x_{j}, t\right)-\dot{y}_{I j}\left(x_{i}, t\right)\right]
$$

where $k_{1}$ and $c_{1}$ are the spring stiffness and viscosity coefficient of the contact between the I-beam and the crossbeam.

Substituting both Eqs. (3) and (4) into Eq. (1) leads to

$$
\begin{aligned}
& E I_{1} y_{I j}^{(4)}(x, t)+m_{1} \ddot{y}_{I j}(x, t)=\sum_{i=1}^{N I} k_{1}\left[y_{c i}\left(x_{j}, t\right)-y_{I j}\left(x_{i}, t\right)\right] \\
& +\sum_{i=1}^{N I} c_{1}\left[\dot{y}_{c i}\left(x_{j}, t\right)-\dot{y}_{I j}\left(x_{i}, t\right)-\frac{P_{n}}{2}[1+\sin (\omega t-\pi / 2)]\left[\delta\left(x-x_{r}\right)+\delta\left(x-x_{l}\right)\right]\right.
\end{aligned}
$$

This is the governing vibration equation for the I-beam. Similarly, we can develop the vibration equation for the crossbeams, and obtain

$$
\begin{aligned}
& E I_{2} y_{c i}^{(4)}(z, t)+m_{2} \ddot{y}_{c i}(z, t)=\sum_{\alpha=1}^{2} k_{2}\left[-y_{c i}\left(z_{0 \alpha}, t\right)\right]+\sum_{\alpha=1}^{2} c_{2}\left[-\dot{y}_{c i}\left(z_{0 \alpha}, t\right)\right] \\
& +\sum_{j=1}^{N J} k_{1}\left[y_{I j}\left(x_{i}, t\right)-y_{c i}\left(z_{j}, t\right)\right]-\sum_{j=1}^{N J} c_{1}\left[\dot{y}_{I j}\left(x_{i}, t\right)-\dot{y}_{c i}\left(z_{j}, t\right)\right]
\end{aligned}
$$

where $E I_{2}$ is the flexural rigidity of the crossbeam, $y_{c i}(z, t)$ the displacement of the $i^{\text {th }}$ crossbeam, $m_{2}$ the mass per unit length. Subscript $\alpha$ indicates the sliding bearing 
support at each end of the crossbeam (assuming they are identical), and $k_{2}$ and $c_{2}$ are the spring stiffness and viscous damping coefficient of the contact between the crossbeam and the sliding bearing. $Z_{0 \alpha}$ is the coordinate of the two sliding bearings under the $i^{\text {th }}$ crossbeam, $Z_{j}$ the coordinate of the contact between the $j^{\text {th }}$ I-beam and the $i^{\text {th }}$ crossbeam in the direction of $Z . N J$ is the total number of I-beams in the system.

Eqs. (5) and (6) can be transformed into a set of second order ordinary differential equations using the Ritz method [22]. The solution of Eq. (5) can be obtained of the form

$y_{I j}(x, t)=\sum_{k=1}^{N L} Y_{I j k}(x) \times q_{I j k}(t)=\sum_{k=1}^{N L} \sin \left(\frac{k \pi}{L_{1}} x\right) \times q_{I j k}(t)$

where $Y_{I j k}(x)$ is the $k^{\text {th }}$ normal mode or characteristic function of the I-beam, and $q_{I j k}(t)$ the regular modal coordinates. $N L$ is the number of vibration modes to be included to ensure numerical convergence of the solution.

Note that Eq. (7) satisfies simple-supported boundary conditions, which differs from the continuous beam model with discrete supports. However, the simple format of eq. (7) can significantly simplify the derivation for the solution. As a first approximation, it is adopted here. And the error introduced by this approximation will be discussed on the difference in the calculated results and the FEA outcome in section 4.1.

Substituting Eq. (7) into (5), and after multiplying both sides by $Y_{I j h}(x) \quad(h=1,2$, $3, \cdots, N L$ ), then taking integration of $x$ from 0 to $L_{1}$, the length of the I-beam, we obtain 


$$
\begin{aligned}
& \int_{0}^{L_{1}} Y_{I j h}(x) Y_{I j k}(x) d x=0 \quad(h \neq k) \\
& \int_{0}^{L_{1}} Y_{I j k}^{2}(x) d x=\frac{L_{1}}{2} \\
& \int_{0}^{L_{1}} Y_{I j k}(x) \frac{d^{4} Y_{I j k}(x)}{d x^{4}} d x=\frac{L_{1}}{2}\left(\frac{k \pi}{L_{1}}\right)^{4}
\end{aligned}
$$

Eq. (5) can then be changed to

$$
\begin{aligned}
& \frac{m_{1} L_{1}}{2} \ddot{q}_{I j m}(t)+E I_{1} \frac{L_{1}}{2}\left(\frac{m \pi}{L_{1}}\right)^{4} q_{I j m}(t)+\sum_{i=1}^{N I} k_{1} Y_{I j m}\left(x_{i}\right) \sum_{k=1}^{N Z} Y_{I j k}\left(x_{i}\right) q_{I j k}(t) \\
& -\sum_{i=1}^{N I} k_{1} Y_{I j m}\left(x_{i}\right) \sum_{b=1}^{N H} Y_{c i b}\left(z_{j}\right) q_{c i b}(t)+\sum_{i=1}^{N I} c_{1} Y_{I j m}\left(x_{i}\right) \sum_{k=1}^{N Z} Y_{I j k}\left(x_{i}\right) \dot{q}_{I j k}(t) \\
& -\sum_{i=1}^{N I} c_{1} Y_{I j m}\left(x_{i}\right) \sum_{b=1}^{N H} Y_{c i b}\left(z_{j}\right) \dot{q}_{c i b}(t)=p(t) \quad(m=1 \text { to } N L)
\end{aligned}
$$

where

$$
p(t)=-\frac{P_{n}}{2}[1+\sin (\omega t-\pi / 2)] \times\left[\sin \left(\frac{k \pi}{L_{1}} x_{l}\right)+\sin \left(\frac{k \pi}{L_{1}} x_{r}\right)\right]
$$

Similarly, Eq. (6) can be derived into

$$
\begin{aligned}
& \frac{m_{2} L_{2}}{2} \ddot{q}_{c i n}(t)+E I_{2} \frac{L_{2}}{2}\left(\frac{n \pi}{L_{2}}\right)^{4} q_{c i n}(t)+\sum_{a=1}^{2} k_{2} Y_{c i n}\left(z_{0 a}\right) \sum_{b=1}^{N C} Y_{c i b}\left(z_{0 a}\right) q_{c i b}(t) \\
& +\sum_{j=1}^{N J} k_{1} Y_{c i n}\left(z_{j}\right) \sum_{b=1}^{N C} Y_{c i b}\left(z_{j}\right) q_{c i b}(t)-\sum_{j=1}^{N J} k_{1} Y_{c i n}\left(z_{j}\right) \sum_{k=1}^{N L} Y_{I j k}\left(x_{i}\right) q_{I j k}(t) \\
& +\sum_{a=1}^{2} c_{2} Y_{c i n}\left(z_{0 a}\right) \sum_{b=1}^{N C} Y_{c i b}\left(z_{0 a}\right) \dot{q}_{c i b}(t)+\sum_{j=1}^{N J} c_{1} Y_{c i n}\left(z_{j}\right) \sum_{b=1}^{N C} Y_{c i b}\left(z_{j}\right) \dot{q}_{c i b}(t) \\
& -\sum_{j=1}^{N J} c_{1} Y_{c i n}\left(z_{j}\right) \sum_{k=1}^{N L} Y_{I j k}\left(x_{i}\right) \dot{q}_{I j k}(t)=0 \\
& \quad(n=1 \text { to } N C)
\end{aligned}
$$

where $Y_{c j b}(x)$ is the $b^{\text {th }}$ normal mode of the crossbeam, and $q_{c i n}(t)$ the regular modal coordinates of the crossbeam. $N C$ is the number of vibration modes of the crossbeam to be included for numerical convergence.

For numerical solutions, the values of the material constants and beam geometric 
values are given in Table.1 where $B$ represents the width of the deck gap.

Table 1. Structural parameters of the expansion unit used in analysis

\begin{tabular}{cccccccccccccc}
\hline Parameters & $B$ & $m_{r 1}$ & $I_{1}$ & $L_{1}$ & $k_{1}$ & $c_{1}$ & $m_{r 2}$ & $I_{2}$ & $L_{2}$ & $k_{2}$ & $c_{2}$ & $E$ \\
\hline Unit & $\mathrm{mm}$ & $\mathrm{kg}$ & $\mathrm{m}^{4}$ & $\mathrm{~m}$ & $\mathrm{~N} / \mathrm{mm}$ & $\mathrm{N} \cdot \mathrm{s} / \mathrm{mm}$ & $\mathrm{kg}$ & $\mathrm{m}^{4}$ & $\mathrm{~m}$ & $\mathrm{~N} / \mathrm{mm}$ & $\mathrm{N} \cdot \mathrm{s} / \mathrm{mm}$ & $\mathrm{MPa}$ \\
Value & $0 \sim 80$ & 76 & $1.2 \mathrm{e}-5$ & 14.75 & $3 \mathrm{e} 4 \sim 1 \mathrm{e} 5$ & $3 \sim 15$ & 80 & $1.6 \mathrm{e}-5$ & 1.05 & $2 \mathrm{e} 5$ & 5 & $2.05 \mathrm{e} 5$ \\
\hline
\end{tabular}

A commercial code SIMULINK in MATLAB [22] is used to solve Eqs. (11) and (13). A single I-beam model was first modeled to understand the basic dynamics of the system. Then assuming a unit design using five I-beams, the dynamic response of the whole unit is calculated with the mode superposition method [23]. Results are discussed in Section 4.

\section{Finite element analysis}

Finite element models were built for a single beam system and multi-beam systems using ABAQUS [24]. The most complicated model includes five I-beams and ten crossbeams, as shown in Fig. 4. 3D beam elements were used for all beams. Boundary conditions are viscoelastic contacts between I-beams and crossbeams, all being defined as spring-damping contacts in the $y$ direction. Crossbeams are supported in $y$ direction by two constrains (item 4 in Fig. 4(b)) which are allowed to slide in the $z$ direction. The sliding constraints are then supported by fixed constraints item 5. Values of the physical parameters assigned in the models are given in Table 1. The dynamic loading is modeled as a moving force pulse of a sine wave at a constant 
speed in the traffic direction, shown in Fig. 3(b) as a time sequence over five I-beams. A mesh sensitivity study shows that a total number of 830 beam elements and 855 nodes provide an appropriate model with good convergence.

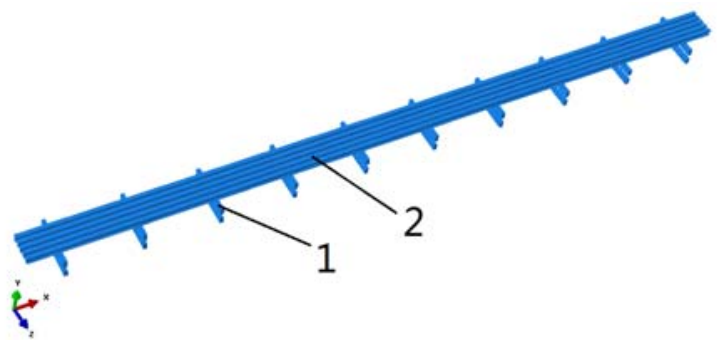

(a) The whole structure

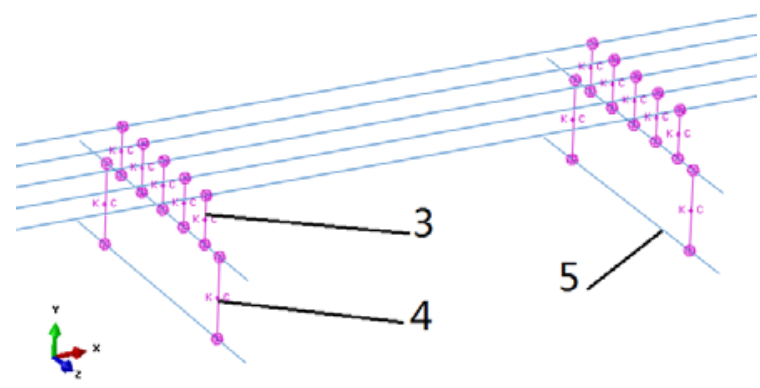

(b) The local structure

Fig.4. The finite element model of expansion joints, 1. Crossbeam, 2. I-beam, 3. Viscoelastic element, 4. Sliding bearing, 5. Fixed constraint

\section{Results and Discussion}

\subsection{Response of a single and multiple I-beams}

Depending on the deck gap, a designer may choose to use a single or several I-beams to reduce the dynamic effect of the traffic. We looked first at a basic model consisted of only one single I-beam with ten supporting crossbeams of equal distance (at the full width of the bridge). The deck gap is $100 \mathrm{~mm}$ and the width of the I-beam is $90 \mathrm{~mm}$. The amplitude of the wheel loading is set at $70 \mathrm{kN}$. The interest here is on the maximum displacement of the I-beam, as the bending stress under the traffic loading may lead to fatigue failure of the system. To ensure result convergence and 
calculation efficiency, $N L$ and $N C$, the numbers of the vibration modes of the I-beam and the crossbeam, respective, need to be specified. Table 2 gives the displacement response of the midpoint of the I-beam in different values of $N L$ and $N C$. It shows that when $N L>25$ and $N C>5$, the displacement does varies. Hence 25 and 5 were chosen in all calculations for $N L$ and $N C$, respectively.

Table 2. Midpoint displacement (in $\mathrm{cm}$ ) of one I-beam system in different NL and NC

\begin{tabular}{rrrrrrr}
$\mathrm{NC}_{N C}^{N L}$ & 10 & 15 & 20 & 25 & 30 & 35 \\
\hline 3 & 2.14 & 2.51 & 2.75 & 2.89 & 2.89 & 2.90 \\
\hline 5 & 2.37 & 2.72 & 2.96 & 3.07 & 3.07 & 3.07 \\
7 & 2.37 & 2.72 & 2.96 & 3.07 & 3.07 & 3.07 \\
\hline
\end{tabular}

Fig. 5 shows displacement histories of the midpoints of the I-beam (Fig. 5(a)) and the No. 6 crossbeam (Fig. 5(b)) respectively when the dynamic loading moves at $80 \mathrm{~km} / \mathrm{h}$. Results of the theoretical model and FEA are very similar, though the latter produces slightly larger magnitude of vibration, particularly for the crossbeam at the first peak. The difference is about $10 \%$. The overall response shows a very strong damping effect in the I-beam with the first peak displacement, marked by A in Fig. 5(a), significantly bigger (thus the highest magnitude value) than the 2nd highest (rebound) for the I-beam. The overall attenuation pattern and period are similar in both the I-beam and the crossbeam.

As mentioned earlier, Eq. (7) is only applicable to simply supported beams. The different boundary conditions in the analytical model and FEA contribute to the differences in their results. Nevertheless, the differences are moderate in the response of both the I-beam and the crossbeam. As can be seen in Fig 5, the overall attenuation is the same, and the frequencies are the same. The only noticeable difference is in the magnitudes which do not differ significantly. With the benefit of much simplified 
derivation for analysis, the adoption of Eq. (7) as the format of solution can be accepted as the first approximation.

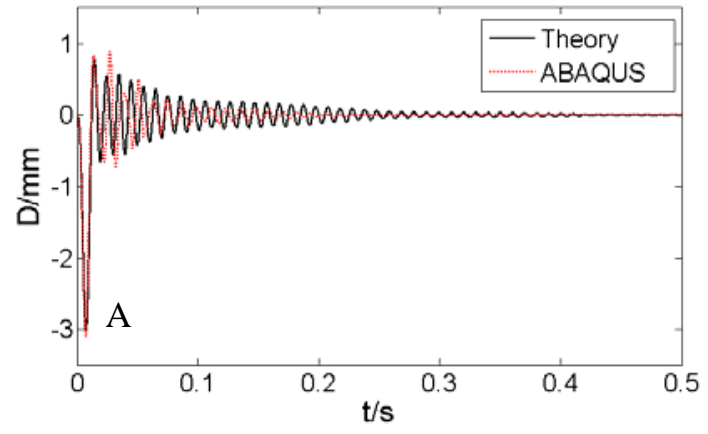

(a) Midpoint of the I-beam

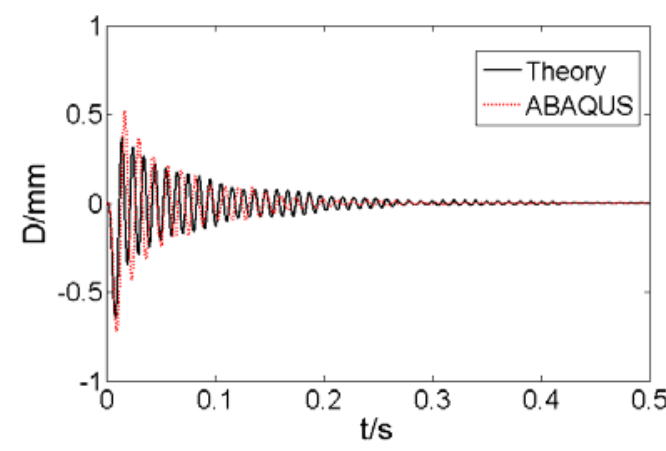

(b) Midpoint of the $6^{\text {th }}$ crossbeam

Fig.5. Dynamic response of one I-beam unit

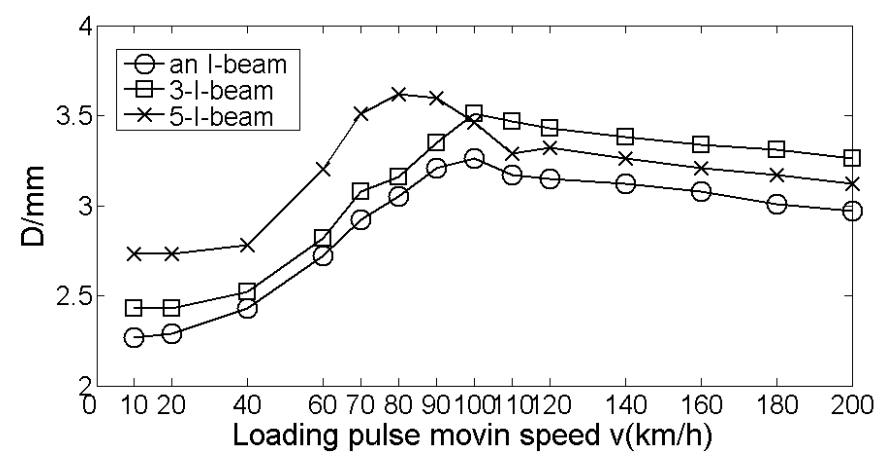

Fig.6. First peak displacement of I-beams vs the speed of the dynamic loading

Two more units were analyzed by FEA, having three I-beams and five I-beams, respectively. Fig. 6 illustrates the first peak displacement (corresponding to reading of A in Fig. 5(a)) in terms of the load moving speed for the units of a single I-beam, 3 and 5 I-beams, respectively. It shows that there exists a particular velocity at which an overall maximum displacement occurs for each unit. For the 5 I-beam unit, the speed is $80 \mathrm{~km} / \mathrm{h}$. And for both the single and 3 I-beam units, the speed is $100 \mathrm{~km} / \mathrm{h}$. This maximum displacement (thus the maximum bending stress) needs to be identified, 
together with the corresponding velocity of the travelling load, for design and maintenance purposes.

\subsection{Full model of an application design (as shown in the Appendix)}

The schematic 2D view of a full expansion unit is shown in the Appendix. The unit has five I-beams and two parallel L-shaped side beams, supported by ten crossbeams. $P_{n}$ is the contact force between the vehicle wheel and the I-beam. Taking the width of the I-beam as $90 \mathrm{~mm}$, the ground contact length of the wheel as $200 \mathrm{~mm}$, and the gap between adjacent I-beams as $40 \mathrm{~mm}, P_{n}$ can be calculated as [12],

$$
P_{n}=\frac{P \times 90}{200-2 \times 40}
$$

Fig.7 shows the trace of the displacements of the midpoint of the $3^{\text {rd }}$ I-beam and the $6^{\text {th }}$ crossbeam (both are in the middle of the system, thus the worst cases), respectively. The overall response is similar to those shown in the previous section (Section 4.1).

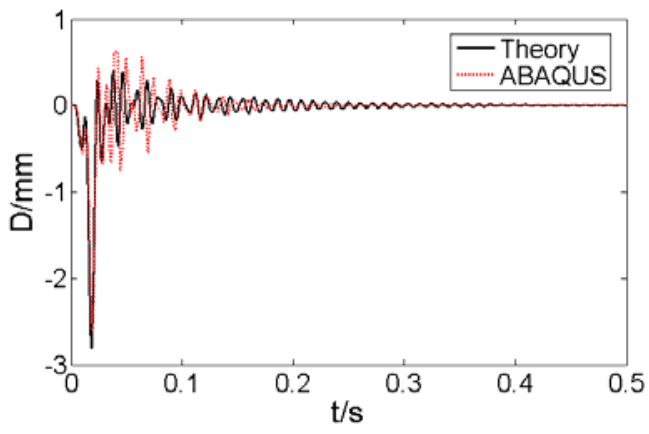

(a) Midpoint of the $3^{\text {rd }}$ I-beam

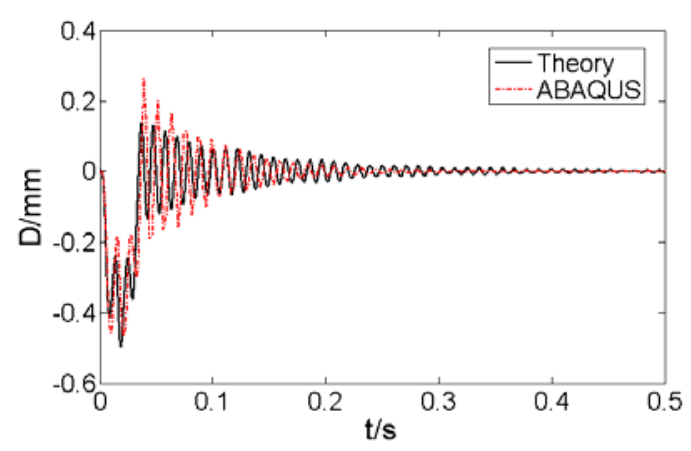

(b) Midpoint of the $6^{\text {th }}$ crossbeam

Fig.7. Displacement response of the full expansion unit

The maximum displacement of the I-beam (Fig. 7(a)) is much larger than that of the crossbeam (Fig. (b)), indicating that the I-beams and its connecting elastic 
elements may fail easily, which is consistent with observations in practice. The damping effect is very strong and the remaining vibration is moderate and attenuated quickly.

As vibration can be controlled to attenuate over a short time, it is possible to optimize structure parameters for improved performance. To achieve this, the parameters of the elastic elements including the impact coefficient of the system, the stiffness and the viscous damping coefficient of the viscoelastic supports are studied in the following sections.

\subsection{The impact coefficient}

The impact coefficient is introduced here as a non-dimensional magnification factor of displacement as defined in Eq. (15),

$$
\alpha=\frac{D_{v}}{D_{0}}
$$

where $D_{v}$ is the maximum displacement of the beam under a moving load at a speed of $v$, and $D_{0}$ the maximum displacement of the beam under the same moving load at a reference speed $v_{0}$.

Using the full model described in section 4.2, the peak displacement of the $3^{\text {rd }}$ I-beam in different load moving speeds is shown in Fig. 8. The influence of the gap width $B$ is also illustrated. Similar to Fig. 6, it shows a maximum value of the peak displacement under a certain velocity.

Bases on Fig. 8, the impact coefficient can be calculated as a function of the load moving speed. Because the peak displacement of the I-beam varies noticeably at 
velocities higher than $10 \mathrm{~km} / \mathrm{h}$, but hardly changes at lower speeds, in calculation, the reference speed was taken as $v_{0}=10 \mathrm{~km} / \mathrm{h}$. In fact, at $10 \mathrm{~km} / \mathrm{h}$, the peak displacement can also be taken as the same of the static loading.

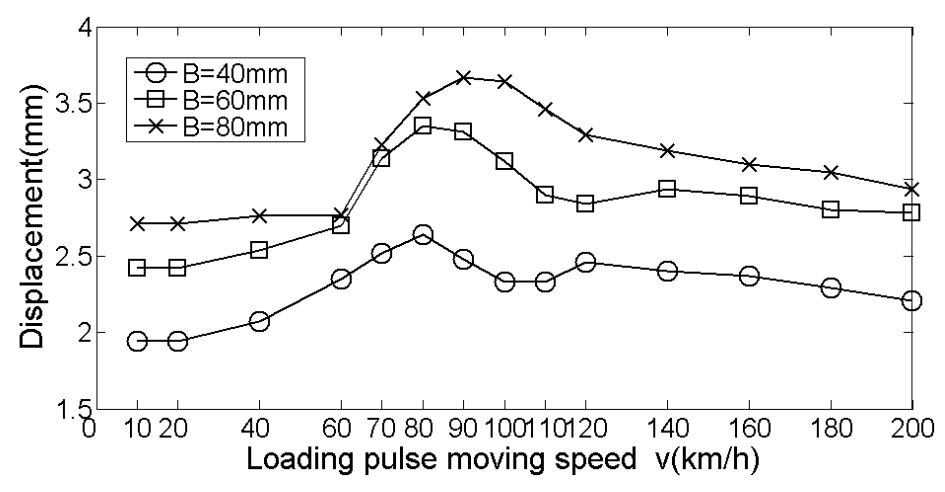

Fig.8. Peak displacement of the $3^{\text {rd }}$ I-beam vs. load moving speed

The calculated impact coefficient of the expansion unit for different joint widths and load moving speeds is shown in Fig. 9. With the increase of speed, $D_{v}$ increases until it reaches the maximum, then reduces. For design purpose, one may do a static analysis, then use Fig. 9 to obtain the maximum dynamic displacement. For instance, for the case $B=40 \mathrm{~mm}$, one can calculate the peak displacement at the speed $80 \mathrm{~km} / \mathrm{h}$ by multiplying 1.36 to the static displacement. We can also draw the conclusion that the maximum bending stress in the I-beam at $80 \mathrm{~km} / \mathrm{h}$ is 1.36 time that of the static one.

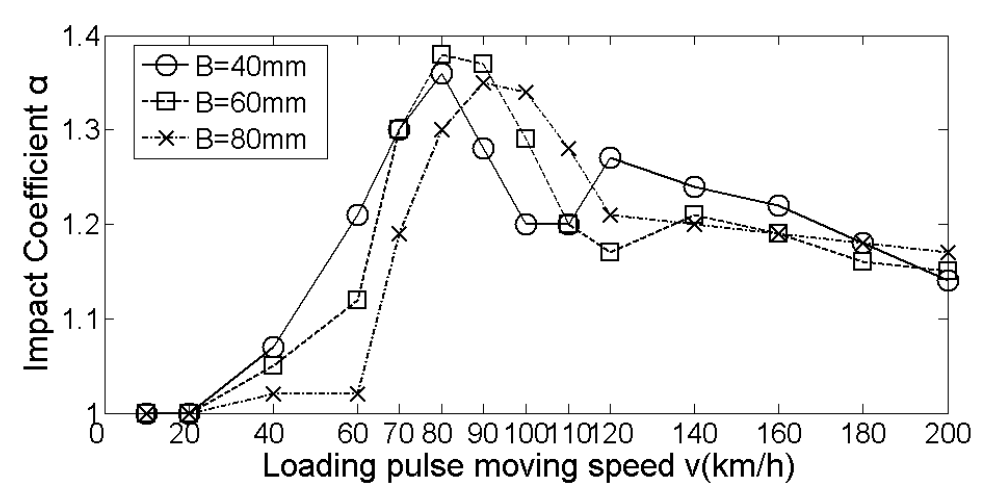

Fig.9. Impact coefficient for increasing vehicle speed 
4.4 The influence of the stiffness and the damping coefficient of the I-beam support ( $k_{1}$ and $c_{1}$ )

Apart from the flexural rigidity of the beams which affects the vibration performance of the system, the viscoelastic supports of the I-beams also play an important role. The supports consist of elastic elements, including rubber pads. They are pre-compressed to control rebounding displacement and to produce very quick attenuation of vibrations (thus the effect of the follow-on dynamic loading does not add on to the overall vibration). Figs. 10 and 12 illustrate the effect of the stiffness and the damping coefficient of the supports for the case in which $B=80 \mathrm{~mm}$ and the load moving speed $v=100 \mathrm{~km} / \mathrm{h}$.

In Fig. 10 where the damping coefficient is assumed to be $c_{1}=5 \mathrm{kNs} / \mathrm{m}$, the maximum displacement of the $3^{\text {rd }}$ I-beam is given, showing a nonlinear relationship in a reducing trend in terms of the stiffness of the support. The rate of the reduction is decreasing. The choice of the $k$ value should satisfy the requirement that the maximum rebounding displacement does not exceeding the applied pre-compression. This is to ensure the contact between the beam and the support always being maintained in compression, and the support is never loaded in tension which may lead to separation failure. For the example shown in Fig. 10, if the pre-compression of the viscoelastic support is $0.2 \mathrm{~mm}, k_{1}$ can be chosen as $90 \mathrm{kN} / \mathrm{mm}$ as the rebound displacement of the support is $0.16 \mathrm{~mm}$. 


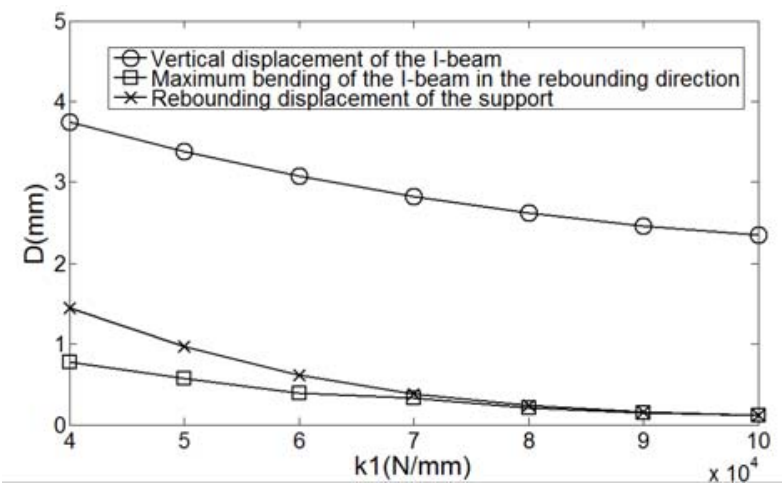

Fig.10. Peak displacement of the $3^{\text {rd }}$ I-beam in different stiffness of the support, $c_{1}=5 \mathrm{Ns} / \mathrm{mm}$.

For attenuation of I-beam vibrations, the concept of the decay time needs to be defined: it is the duration from the moment the dynamic loading leaves the I-beam to that when the displacement amplitude of the I-beam is no more than $0.01 \mathrm{~mm}$, as shown in Fig.11. For the case $B=80 \mathrm{~mm}, v=100 \mathrm{~km} / \mathrm{h}$, and $k_{1}=80 \mathrm{kN} / \mathrm{mm}$, the decay time shows a rapidly reducing trend in terms of the damping coefficient of the support, as illustrated by Fig. 12.

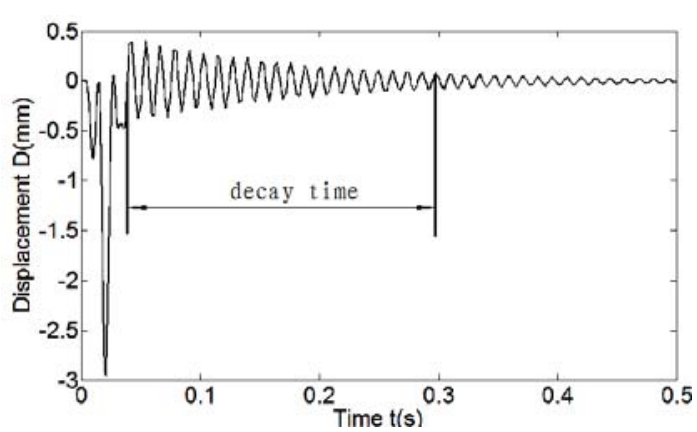

Fig.11. Dynamic response of I-beam for the definition of the decay time

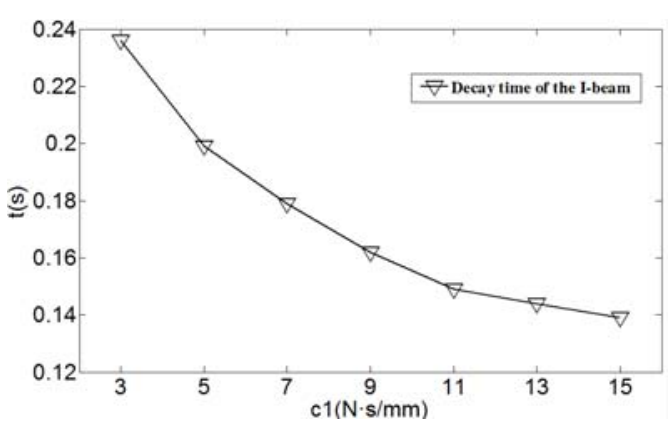

Fig.12. Decay time in different damping coefficient of the support, $k_{1}=80 \mathrm{kN} / \mathrm{mm}$

To ensure the system is attenuated before the arrival of the following dynamic loading, the decay time should satisfy

$t \leq \frac{d}{v}$

where $d$ is the distance between the dynamic loading, such as the wheel base which is 
the distance between the front and rear wheels of a vehicle, and $v$ the travelling speed of the vehicle. Based on this, the value of $c_{1}$ can be chosen. For instance, from Fig. 12, for a lorry with a wheel base of $5 \mathrm{~m}$ travelling at $100 \mathrm{~km} / \mathrm{h}$, the decay time is $0.18 \mathrm{~s}$ and the damping coefficient of the elastic element can be calculated as $7 \mathrm{kNs} / \mathrm{m}$.

\section{Conclusions}

The dynamic response of a continuous beam with discrete viscoelastic supports was studied using a theoretical analysis and finite element simulations, to describe the vibration behavior under a moving sinusoidal pulse. Numerical results of the theoretical analysis agree reasonable well with those of the finite element modeling. Single and multi-beam units, and a practical design case were studied. Several system parameters were explored for design considerations. The following conclusions can be obtained:

- The bending displacement of I-beams on top is substantially larger than that of the supporting crossbeams, rendering the I-beams prone to fatigue failure under vibration.

- The peak displacement of the I-beams is sensitive to the load moving (traffic) speed. A maximum value of the peak displacement occurs at a particular velocity (Figs. 6 and 8). This maximum value and the corresponding velocity need to be identified to check for the maximum bending stress for design and maintenance.

- An impact coefficient is introduced, allowing the maximum dynamic bending displacement at different speeds being calculated from its static displacement. 
- The selection of the damping coefficient of the viscous supports can be based on the decay period corresponding to the speed of the moving pulse (eg. the speed limit on the bridge).

Though the theoretical model and the finite element analysis have shown good agreement, comparison with experimental measurement is yet to be achieved. This remains as the scope for future work.

\section{Acknowledgments}

This study was supported by the Fundamental Research Fund of the Central Universities (2010ZT03). The authors are grateful to the reviewers for their contribution to the improvement of this paper.

\section{References}

1. Zhao HP, Modern bridge expansion device, China Communications Press, 2008:1-73

2. Li YH etc. The practical handbook of highway bridge expansion device, China Communications Press, 2007:1-68.

3. Zhao HP, Primary investigation to large displacement expansion joints for partial bridges in China. Technology of Highway and Transport, 2006 (5):76-80.

4. Guerreiro H., Campos-Rebelo R., Gomes L. Bridge expansion joints monitoring system. Intelligent Engineering Systems (INES), 2011:11-16.

5. Kim SY, Gong SL, Lee JW. Joint optimization of link weight and link capacity expansion in communication networks. Information and Communication Technology Convergence (ICTC), 2010:125-126.

6. Wang TL, Li QN. Effect of expansion joint on seismic response of curved ramp bridge. Electric Technology and Civil Engineering (ICETCE), 2011:5904-5907.

7. Yue X, Jiang B, Wang X, Application of modular expansion joints on bridge. 
Communications Science and Technology Heilongjiang,2011(3):55-56.

8. Wang LC et al, Experimental research on fatigue testing of modular bridge expansion joint, China Civil Engineering Journal, 2004 (37):44-49.

9. Dexter RJ, Osberg CB, Mutziger MJ. Design, specification, installation, and maintenance of modular bridge expansion joint systems, Journal of Bridge Engineering, 2001(6):529-538.

10. Coelho BZ et al. Dynamics of modular expansion joints: The Martinus Nijhoff Bridge, Engineering Structures, 2013(48): 144-154.

11. Dexter RJ, Mutziger MJ, Osberg CB, Performance testing for modular bridge joint systems, Tech.Rep., National Cooperative Highway Research Program-Report 467;2002.

12. Steenbergen M. Dynamic response of expansion joints to traffic loading, Engineering Structures, 2004 (16):1677-1690.

13. Roeder C. Fatigue and dynamic load measurements on modular expansion joints, Construct Build Mater 1988(12):143-150.

14. Chaallal O, Sieprawski G, Guizani L. Fatigue performance of modular expansion joints for bridges, Canadian Journal of Civil Engineering, 2006(33):921-932.

15. Crocetti R, Edlund B, Fatigue performance of modular bridge expansion joints, Journal of Performance of Constructed Facilities, 2003(17):167-176.

16. Ghimire JP, Matsumoto Y, Yamaguchi H, Kurahashi L, Numerical investigation of noise generation and radiation from an existing modular expansion joint between prestressed concrete bridges, Journal of Sound and Vibration, 2009(328): 129-147.

17. Yu TX, Stronge WJ, Large deflection of a rigid-plastic beam-on-foundation from impact, Int. J. Impact Engng. 9(1), 115-126, 1990.

18. Chen XW, Yu TX and Chen YZ, Modeling of elastic-plastic beam-on-foundation, in Advances in Engineering Plasticity, Part 2 (ed. T.X. Yu, Q.P. Sun and J.K. Kim), Key Engineering Materials, 177-180, 727-732, 2000.

19. Chen XW and Yu TX, Elastic-plastic beam-on-foundation under quasi-static loading, Int. J. Mech. Sci. 42, 2261-2281, 2000.

20. Zhou FH, Yu TX and Yang LM, Elastic behavior of ring-on-foundation, Int. J. Mech. Sci. 54(1), 38-47, 2012.

21. Zhai WM, Vehicle-Track Coupling Dynamics, Beijing: Science Press, 2007:1-128. 
22. MATLAB, Mathworks, 3 Apple Hill Drive, Natick, MA 01760-2098, USA

23. MacDonald JK, Successive Approximations by the Rayleigh-Ritz Variation Method, Phys. Rev. 43(10), 830-833, 1933.

24. Abaqus, Dassault Systèmes, 10 rue Marcel Dassault, CS 40501, 78946 Vélizy Villacoublay Cedex, France 


\section{Appendix Details of a bridge expansion unit used as the model in the analysis}

The modular expansion unit consists of five I-beams (sometimes termed centre beams) and two side beams positioned perpendicular to the bridge axis. The I-beams are supported by crossbeams. The side beams are fixed on the joist box at the end of the bridge gaps. The crossbeams are supported at the ends by the joist boxes with sliding bearings and the pre-stress element to allow for the expansion and contraction of the structure. Brackets, pre-stress and pressing elements are used to coordinate the sliding motion and the deformation of the crossbeam. The vertical stiffness of the pre-stress and pressing elements can be treated as spring-damper supports of the beams. Waterproof rubber shields are used to prevent water and debris/dirt dropping into the system and have little influence on the mechanical performance of the system.

Fig. A1 shows the schematic view of a design of an expansion joint unit.

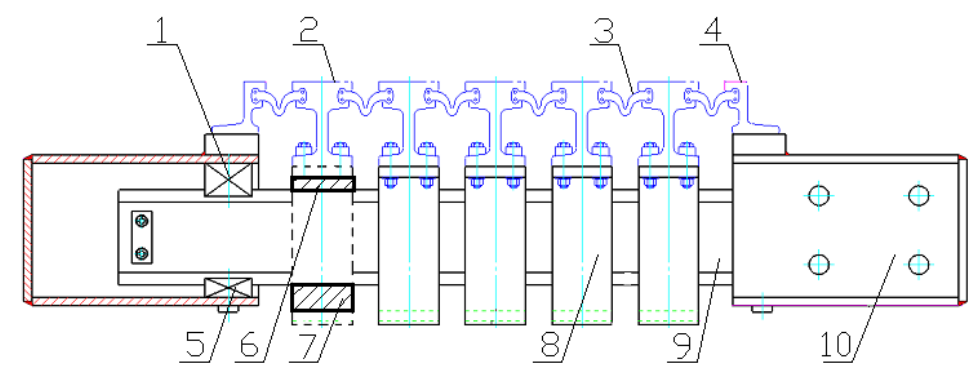

Fig.A1. Schematic view of ZL480 expansion joints

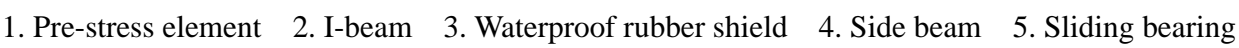
6. Elastic element $\quad$ 7. Pressing element $\quad$ 8. Bracket 9. Crossbeam 10. Joist box 